\title{
Desenvolvimento de um Simulador Computacional para Problemas Globais de Reatores Nucleares
}

\author{
Luiz Fernando Trindade Santos Hermes Alves Filho \\ Universidade do Estado do Rio de Janeiro - Departamento de Modelagem Computacional \\ 28625-570, Campus Regional de Nova Friburgo, RJ \\ E-mail: 1luizfernandotrindade@gmail.com \\ halves@iprj.uerjbr
}

\section{RESUMO}

Nos dias de hoje, as áreas de pesquisas que mais se desenvolvem, relacionadas às engenharias, estão diretamente vinculadas aos vários aspectos da modelagem computacional. É uma metodologia moderna de investigação que requer a aplicação de algoritmos computacionais sofisticados e eficientes.

$\mathrm{Na}$ área de Engenharia Nuclear, na simulação de problemas envolvendo cálculos globais de reatores nucleares, procuramos desenvolver algoritmos computacionais que possam descrever com grande precisão a distribuição de potência nuclear, a variação de reatividade no núcleo do reator, o cálculo de queima do combustível nuclear, problemas de recarga de reatores nucleares etc [1]. Essas modelagens computacionais se justificam pois estão diretamente relacionadas com o funcionamento de um reator nuclear, opção energética viável para a atenuação de problemas relativos a questão do aquecimento global.

Neste trabalho, propomos o desenvolvimento de uma modelagem computacional para o cálculo de distribuição de potência nuclear e o fator de multiplicação efetivo $\left(\mathrm{k}_{\mathrm{eff}}\right)$ [1], domínio unidimensional, nêutrons monoenergético e regime estacionário, usando a equação de transporte de nêutrons, na formulação de ordenadas discretas $\left(\mathrm{S}_{\mathrm{N}}\right)$ [2-3]. Esse problema não possui solução analítica sendo necessária uma abordagem numérica dessa solução. O método usado para a discretização da variável espacial do fluxo angular de nêutrons é o método de malha grossa espectro-nodal híbrido SD-SGF [4], c.f., spectral Diamond-spectral Green's function. Esse hibridismo é necessário para garantirmos a estabilidade numérica dos algoritmos computacionais desenvolvidos. $\mathrm{O}$ método SD [4] é usado em regiões multiplicativas (onde há a presença do fenômeno da fissão) e o método SGF [5] é utilizado nas regiões não-multiplicativas (onde não há fissão) do domínio material de interesse. Na solução das equações de transporte de nêutrons discretizadas de balanço espacial $\left(S_{N}\right)$, com condições de contorno apropriadas [1,2] e as equações auxiliares SD-SGF, usamos o método iterativo NBI [5], cf., "Onenode block inversion" para os cálculos dos fluxos angulares (iterações internas) e o tradicional método das potências (iterações externas) [6-7], para o cálculo do fator de multiplicação efetivo ( $\left.\mathrm{k}_{\text {eff }}\right)$. Os resultados dessas simulações serão comparados com o tradicional método de malha fina DD, cf., Diamond Difference [2,4], convencionalmente usado nesse tipo de simulação.

Os algoritmos de solução dos problemas modelados serão implementados num simulador computacional em linguagem MatLab, o mesmo que foi utilizado para gerar os resultados do trabalho da referência [8], sendo que aqui os problemas desenvolvidos foram de blindagem de nêutrons, ou seja, problemas de fonte-fixa, também de grande interesse no funcionamento de reatores nucleares de potência.

Palavras-chave: Modelagem Computacional, Teoria de Transporte de Nêutrons, Ordenadas Discretas 


\section{Referências}

[1] Duderstadt J.J. e Hamilton L.J., Nuclear Reactor Analysis, John Wiley \& Sons, New York (1976).

[2] Lewis E.E. e Miller W.F.Jr., Computational Methods of Neutron Transport, American Nuclear Society, La Grange Park, Illinois, 1993.

[3] Alcouffe, R.E. e O'Dell, R.D., Transport Calculations for Nuclear Reactors, CRC Handbook of Nuclear Reactors Calculations, Vol. 1, CRC Press. Inc, Florida, 1986.

[4] de Abreu, M. P., "Métodos Determinísticos Livres de Aproximações Espaciais para a Solução Numérica Dominante de Problemas de Autovalor Multiplicativo na Formulação de Ordenadas Discretas da Teoria do Transporte de Nêutrons". Tese de D.Sc., COPPE/UFRJ, Rio de Janeiro, RJ, Brasil,1996.

[5] Barros R.C., A Spectral Nodal Method for the Solution of Discrete Ordinates Problems in one and two Dimensional Cartesian Geometry. Ph.D. dissertation, The University of Michigan, Ann Arbor, Michigan, (1990).

[6] Nakamura S., Computational Methods in Engineering and Science, John Wiley \& Sons, New York, 1977.

[7] Fladmark, G. E., IAEA-Numerical Reactor Calculations, Vienna, Jan. 17-21, Proc., IAEA-SM-154/20, 1972.

[8] Reis R. B., Filho H. A.; Zani J. H. Modelagem Computacional para Cálculos de Blindagem de Nêutrons em Problemas de Engenharia Nuclear. In: Congresso Nacional de Matemática Aplicade e Computacional, XXXIII CNMAC. São Paulo. Águas de Lindoia, SP: Publicação SBMAC, p. 32-38, 2010. 